\title{
Molecular Mechanism of Atopic Dermatitis Induction Following Sensitization and Challenge with 2,4-Dinitrochlorobenzene in Mouse Skin Tissue
}

\author{
JiYoun Kim, JaeHee Lee, SoJung Shin, AhRang Cho and Yong Heo \\ Department of Occupational Health, Catholic University of Daegu, Gyeongsan, Korea
}

\begin{abstract}
Laboratory animal models have been developed to investigate preventive or therapeutic effect of medicinal products, or occurrence or progression mechanism of atopic dermatitis (AD), a pruritic and persistent inflammatory skin disease. The murine model with immunologic phenomena resembling human AD was introduced, which demonstrated skewedness toward predominance of type- 2 helper T cell reactivity and pathophysiological changes similar as human AD following 2,4-dinitrochlorobenzene (DNCB) sensitization and challenge. Molecular mechanism on the DNCB-mediated AD was further evaluated. Skin tissues were collected from mice treated with DNCB, and each tissue was equally divided into two sections; one for protein and the other for mRNA analysis. Expression of filaggrin, an important protein for keratinocyte integrity, was evaluated through SDS-PAGE. Level of mRNA expression for cytokines was determined through semi-quantitative reverse transcriptase polymerase chain reaction. Expression of filaggrin protein was significantly enhanced in the mice treated with DNCB compared with the vehicle (acetone : olive oil $=4: 1$ mixture) treatment group or the normal group without any treatment. Level of tumor necrosis factor-alpha and interleukin-18 mRNA expression, cytokines involved in activity of type-1 helper $\mathrm{T}\left(\mathrm{T}_{\mathrm{H}} 1\right)$ cell, was significantly downregulated in the $\mathrm{AD}$ group compared with other control groups. These results suggest that suppression of $\mathrm{T}_{\mathrm{H}} 1$ cell-mediated immune response could be reflected into the skin tissue of mice treated with DNCB for AD induction, and disturbance of keratinocyte integrity might evoke a compensatory mechanism.
\end{abstract}

Key words: Atopic dermatitis, Murine model, Filaggrin, IL-18, TNF $\alpha$, Gene expression

\section{INTRODUCTION}

Human atopic dermatitis (AD) is the most prevalent inflammatory skin disease characterized with progressive inflammation resulting in pruritic eczematous skin lesions in children and could be recurrent in adulthood (1). Prevalence of $\mathrm{AD}$ is approximately $10 \%$ in developed countries and continuously increasing to $20 \%$ in developing countries (2). AD is considered a multifactorial disease involved

Correspondence to: Yong Heo, Department of Occupational Health, Catholic University of Daegu, 13-13 Hayang-ro, Hayangeup, Gyeongsan-si, Gyeongbuk 38430, Korea

E-mail:yheo@cu.ac.kr

This is an Open-Access article distributed under the terms of the Creative Commons Attribution Non-Commercial License (http:// creativecommons.org/licenses/by-nc/3.0) which permits unrestricted non-commercial use, distribution, and reproduction in any medium, provided the original work is properly cited. with genetic risk, environmental factors, and immune dysregulation. Concerning immune abnormalities, skewedness toward upregulation of type- 2 helper $\mathrm{T}$ cell $\left(\mathrm{T}_{\mathrm{H}} 2\right)$ has been known as a representative phenomenon, which could result in elevation of serum IgE level, enhancement of interleukin (IL)-4, IL-5, or IL-13 production, but downregulation of IL-12 or interferon-gamma (IFN $\gamma$ ) production from helper T cells (3).

Even though $\mathrm{AD}$ is considered a human skin disease, canine $\mathrm{AD}$ is also reported (4). Laboratory animal models have been developed for investigating pathophysiological mechanism of AD (5-9). The animal models are categorized into three types; spontaneous appearance of $\mathrm{AD}$-like cutaneous symptoms, induction of $\mathrm{AD}$ through genetic mutations, and induction $\mathrm{AD}$ related immune alterations through chemical or microbiological application (10). Concerning the spontaneous model, $\mathrm{NC} / \mathrm{Nga}$ mouse model has been extensively evaluated. NC/Nga mice were reported 
to develop spontaneous dermatitis resembling human $\mathrm{AD}$ on their faces under conventional environmental conditions (5). Regarding transgenic animal model, type-2 cytokines such as interleukin (IL)-4, IL-13, or pro-inflammatory cytokines including IL-18 have been target genes for the genetic mutations in mice (6-8). Meanwhile, Staphylococcus aureus application on dorsal skin of DS-Nh hairless mouse was reported to induce pathophysiological outcomes in skin and immune system similar with $\mathrm{AD}(9)$. We also reported a murine model for experimental AD induction. The model was reported to result in upregulation of serum IgE, and concomitant increase in serum IgG1 and decrease in IgG2a in mice sensitized and challenged with DNCB (11). In addition, IL-4 production from in vitro stimulated splenic $\mathrm{T}$ cells was enhanced whereas IFNg production was downregulated in mice applied with DNCB than the control mice. Furthermore, relatively upregulated $\mathrm{IgG} 1$ production was reported compared with IgG2a production from in vitro stimulated splenic B cells in the DNCB-applied mice (10). Production of IL-13, a key player for differentiation of naïve dendritic cell (DC) to DC2 phenotype (12), was significantly higher from axillary lymph node $\mathrm{T}$ cells in the mice treated with $\mathrm{DNCB}$ for $\mathrm{AD}$ induction than the control mice, which accompanied with increased number of dendritic cell. Infiltration of inflammatory cells in dermis and epidermal hyperplasia was apparent in the DNCB-applied mice (13).

Considering the predominance of $\mathrm{T}_{\mathrm{H}}$ 2-mediated humoral and cellular immune reactivity and the histopathologic findings characterized in our animal model, the present study was undertaken to further investigate the DNCBmediated modulation of core protein expression in skin tissue from mice induced with AD. Expression of filaggrin, an essential protein for skin integrity, was examined at protein level. In addition, reverse transcriptase polymerase chain reaction (RT-PCR) was performed to evaluate mRNA expression of various cytokines including IL13, IL-18, and tumor necrosis factor-alpha (TNF $\alpha)$ in skin tissues of mice treated with DNCB for AD induction and compared with control mice.

\section{MATERIALS AND METHODS}

Animals and atopic dermatitis induction. Specific pathogen free 4 week old male BALB/c mice were purchased from an animal supplier (Orient Bio, Seongnam, Korea). Mice were housed in laminar flow cages and were maintained on irradiated food and autoclaved water. Mice were acclimatized for $4 \sim 5$ days before initiation of experimentation. All of our animal maintenance and experimental procedures were approved by Catholic University of Daegu's IACUC (IACUC-2010-33). The detailed method for induction of atopic dermatitis like immune alterations is described at our previous reports $(10,11,13)$. Briefly,
$\mathrm{BALB} / \mathrm{c}$ mice were sensitized twice with $100 \mathrm{~mL}$ of $1 \%$ DNCB (Sigma, Saint Louis, MO, USA) or vehicle (acetone : olive oil = 4:1 mixture; AOO) in a week and challenged twice with $100 \mathrm{~L}$ of $0.2 \%$ DNCB or the vehicle at the following week on dorsal skin shaved $(2 \times 4 \mathrm{~cm})$. Mice were sacrificed at 4 days after the second DNCB or vehicle challenge, followed by collection of skin tissues. Each skin tissue was equally divided into two sections; one for protein homogenization and the other for RNA extraction. Number of skin tissues collected were 45 for the AD group, 21 for the vehicle group, and 10 for the normal mice without any treatment.

\section{Homogenization of skin tissues and Western blotting.}

Skin tissues were homogenized in extraction buffer [20 $\mathrm{mM}$ Tris (pH 8, Bio Basic Inc., Markham, Canada), 100 $\mathrm{mM} \mathrm{NaCl}, 2 \mathrm{mM}$ disodium ethylene diamine tetraacetic acid (Sigma), 1\% Igepal CA-630 nonionic detergent (Sigma), 1\% protease inhibitor cocktail (Sigma)] and sonicated for $10 \mathrm{~min}$. Then samples were centrifuged for 30 $\min \left(16,000 \times \mathrm{g}, 4^{\circ} \mathrm{C}\right)$ and the resulting supernatants were collected and frozen at $-80^{\circ} \mathrm{C}$ until use. Concentrations of homogenate protein were determined using a BCA protein assay kit (Thermo Scientific, Rockford, IL, USA). For Western blotting (14), $30 \mu \mathrm{g}$ of protein were separated on $10 \%$ sodium dodecyl sulfate polyacrylamide gel (SDSPAGE) and transferred onto a nitrocellulose membrane (pore size $0.45 \mu \mathrm{m}$, Bio-Rad, Hercules, CA, USA). Membranes were blocked with $5 \%(\mathrm{w} / \mathrm{v})$ nonfat dry milk (Sigma) and $0.3 \%$ Tween 20 in PBS (PBS-T) overnight at $4^{\circ} \mathrm{C}$ on a rocking platform, and then incubated at room temperature for $1 \mathrm{hr}$ in PBS-T containing $5 \%(\mathrm{w} / \mathrm{v})$ nonfat dry milk and anti-filaggrin antibody $(0.2 \mathrm{mg} / \mathrm{mL}, 1: 200$ dilution, Santa Cruz, Dallas, TX, USA). Membranes were washed two times for $5 \mathrm{~min}$ each with tris buffered saline (TBS), and then washed two times for $5 \mathrm{~min}$ each with $0.05 \%$ Tween 20 in TBS (TBS-T), and incubated at room temperature for $1 \mathrm{hr}$ in TBS-T containing goat anti-rabbit IgG HRP conjugate (1 : 5000 dilution, Santa Cruz). Antiglyceraldehyde-3-phosphate dehydrogenase (GAPDH) antibody (Santa Cruz, $1: 200$ dilution in blocking buffer) was also used as an internal loading control. After washing three times for $5 \mathrm{~min}$ each with TBS-T and rinsing three times for 5 min each with TBS, chemiluminescence detection was performed using a chemiluminescence kit (Amersham ECL Western Blotting Kit, GE Healthcare Life Sciences, Pittsburgh, PA, USA). The blot was visualized with UVITEC Cambridge imaging system (Union Lane, UK).

RNA extraction and semi-quantitative RT-PCR. RTPCR was performed to detect mRNA of various cytokines including IL-13, IL-18, and TNF- $\alpha$ in skin tissues of mice. Semi-quantitative RT-PCR methods are described else $(15,16)$. Total RNA was extracted using the RNA 
extraction buffer RNAzol (Invitrogen, Rockford, IL, USA). A Transcriptor First Strand cDNA Synthesis kit (Roche, Mannheim, Germany) was used for RT-PCR. After termination of the cDNA reaction through heating for $5 \mathrm{~min}$ at $99^{\circ} \mathrm{C}$, the PCR reaction was carried out according to the supplier's instructions to amplify reverse-transcribed cDNA using primer templates of the cytokines and internal control glyceraldehyde-3-phosphate dehydrogenase (GAPDH). Primers were synthesized by a company (Bionics, Seoul, Korea) as followings: IL-13 sense, 5'-GCCAGCCCACAGTTCTACAGC-3' and IL-13 anti-sense, 5'-GTGATGTTGCTCAGCTCCTCA-3'; IL-18 sense, 5'-ACTGTACAACCGGAGTAATACGG-3' and IL-18 anti-sense, 5'TCCATCTTGTTGTGTCCTGG-3'; TNF $\alpha$ sense, 5'AACTAGTGGTGCCAGCCGAT-3' and TNF $\alpha$ ant-sense, 5'-CTTCACAGAGCAATGACTCC-3'; GAPDH sense, 5'-CTGGTGCTGAGTATGTCGTG-3' and GAPDH antisense 5'-CAGTCTTCTGAGTGGCAGTG-3. A volume of $20 \mu \mathrm{L}$ of each PCR Product was electrophoresed in $1.5 \%$ agarose gel in Tris acetate/EDTA buffer and the gels were stained with ethidium bromide for visualization of amplified cDNA. Densitometry analysis was carried out with Scientific Imaging Systems (Eastman Kodak, New York, NY, USA) and Kodak 1D Image Analysis Software (Eastman Kodak).

Statistical analyses. Statistical significances on the expression of filaggrin or cytokines between the groups were tested using SigmaStat (SPSS, Chicago, IL, USA) by Student's $t$ test or Mann-Whitney Rank Sum test depending on normality of data. When $p$ value was below 0.05 , it was considered statistically significant.

\section{RESULTS}

Significantly higher induction of filaggrin protein expression in mice with atopic dermatitis. Since filaggrin is considered a key component for epidermal structure and function (17), level of filaggrin protein expression was examined following the atopic dermatitis induction and compared with the AOO vehicle control or the normal control without any treatment (Fig. 1A). Expression of filaggrin protein was quantitated in relative densitometric units compared with GAPDH expression level for each skin tissue homogenate. Relative density of filaggrin vs GAPDH was significantly higher $(1.70 \pm 0.11)$ for the skin tissues from mice with atopic dermatitis than the vehicle control $(1.02 \pm 0.05)$ or the normal mice $(1.01 \pm 0.05)$ (Fig. 1B). No difference was observed between the vehicle control and the normal mice.

Significantly lower expression of IL-18 and TNF- $\alpha$ mRNA transcript in mice with atopic dermatitis. Since IL-13 is known to be an important player for systemic
(A)

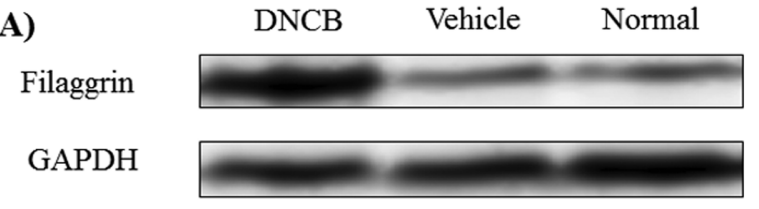

(B)

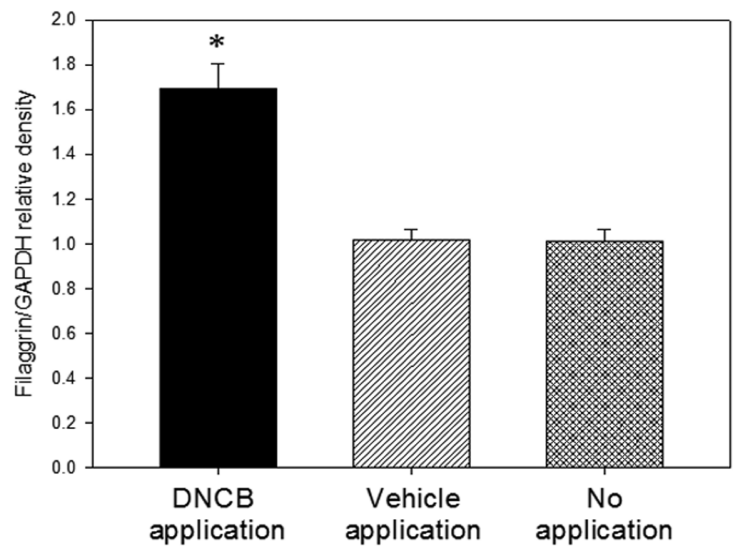

Fig. 1. Filaggrin expression in skin tissue is enhanced in the mice treated with DNCB for atopic dermatitis induction compared with the vehicle control or the normal mice. Representative figure for Western blotting is shown at the top (A). Relative expression density (fold induction) of filaggrin protein was obtained through dividing each filaggrin density value by the value of each GAPDH, an internal loading control. Data were obtained from 45, 21, and 10 mice for the DNCB application, the vehicle control, and the normal control, respectively, and expressed as means \pm standard errors (B). The asterisk $\left(^{*}\right)$ indicates significantly higher $(p<0.05)$ than the vehicle group and the non-treatment normal control group.

$\mathrm{T}_{\mathrm{H}} 2$-mediated immune response, which is a central dogma for atopic dermatitis occurrence (18), mRNA expression level of IL-13 was evaluated in skin tissues after the atopic dermatitis induction (Fig. 2A, left). IL-18 mRNA expression was also examined (Fig. 2A, middle) since IL-18 is one of participants in $\mathrm{T}_{\mathrm{H}} 1$-mediated immune responses including allergic contact dermatitis, a typical type-4 hypersensitivity reaction (19). In addition, TNF- $\alpha$ was also investigated (Fig. 2A, right) because this cytokine is a proinflammatory cytokine expressed in skin keratinocyte and other cells (20), resulting in modulation of skin inflammatory progression. Expression of cytokine mRNA transcript was normalized against the GAPDH value for each skin tissue. Relative mRNA expression of IL-18 vs GAPDH was significantly lowered $(0.31 \pm 0.02)$ for the skin tissues from mice with atopic dermatitis than the vehicle control $(0.50 \pm 0.03)$ or the normal mice $(0.53 \pm 0.05)$ (Fig. 2B). Relative mRNA expression of TNF- $\alpha$ vs GAPDH was significantly lowered $(0.46 \pm 0.02)$ for the skin tissues from mice with atopic dermatitis than the vehicle control $(0.58 \pm 0.03)$, but no difference with the normal mice $(0.54 \pm 0.11)$. IL-13 mRNA expression levels were shown similar among the groups. 
(A)

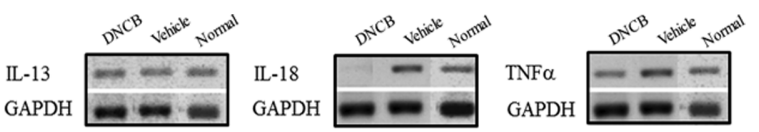

(B)

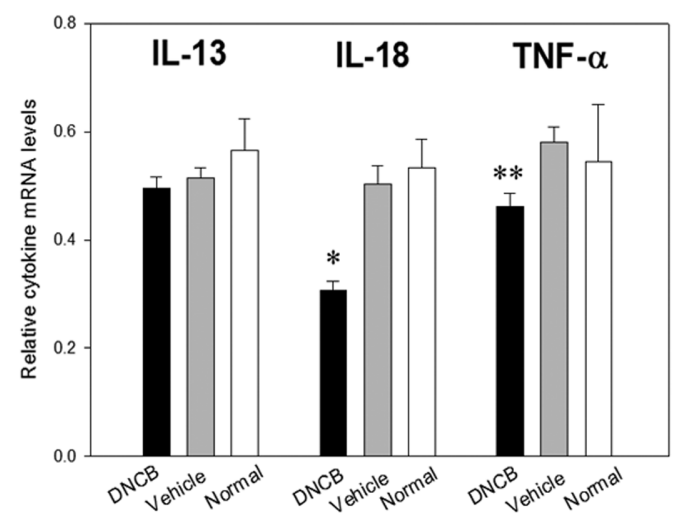

Fig. 2. Expression of IL-18 and TNF- $\alpha$ mRNA transcript is downregulated in the mice treated with DNCB for atopic dermatitis induction compared with the vehicle control or the normal mice. Representative figure for RT-PCR is shown at the top (A). Expression level of each cytokine mRNA was normalized against the value of each GAPDH mRNA expression. Data were obtained from 45, 21, and 10 mice for the DNCB application, the vehicle control, and the normal control, respectively, and expressed as means \pm standard errors (B). The asterisk (*) indicates significantly lower $(p<0.05)$ than the vehicle group and the non-treatment normal control group, and the asterisk $\left(^{* *}\right)$ indicates significantly lower $(p<0.05)$ than the vehicle group.

\section{DISCUSSION}

Filaggrin gene $(F L G)$ null mutation has been reported to be well correlated with susceptibility to or pathogenesis of atopic dermatitis in human subjects $(2,17,22)$. Epidermal expression of filaggrin protein has a function to keep a structural strength of stratum corneum resulting in protection of epidermal integrity against water loss and microbial or chemical penetration (17,21-23). Therefore, the fact that we demonstrated not the down-regulation but the up-regulation of filaggrin protein expression in the skin of mice with atopic dermatitis through DNCB application (Fig. 1) is unexpected. A possible explanation might be that production of filaggrin protein could be enhanced for compensation of reduced filaggrin level at early stage of atopic dermatitis induction in the DNCB-mediated atopic dermatitis induction model. Since the model is considered to reflect the predominance of $\mathrm{T}_{\mathrm{H}}$-2-mediated response in acute phase of atopic dermatitis progression $(10,11,13)$, a feed-back mechanism as yet unknown could be operated as early as possible following DNCB sensitization and challenge for preventing loss of skin integrity. The compensation potential was suggested in a human study (24), in that some FLG mutation carriers are able to compen- sate for reduced amounts of filaggrin in their skin. Whereas, if more expression of filaggrin could lead to more generation of filaggrin breakdown products which could result in greater histamine release, the increased expression of filaggrin protein may be an indicator for aggravation of atopic dermatitis pathogenesis. This speculation has been made for skin tissues treated with 2,3,7,8-tetrachlorodibenzi- $p$ dioxin (25) or in chronic idiopathic urticarial, another type of human chronic inflammatory skin disease (26). Concerning the result on filaggrin protein expression, the immune alterations in the present murine model, which induces pathophysiological changes resembling human atopic dermatitis, may not be implied with genetic alteration leading to some FLG mutation.

IL-18, previously known as IFN $\gamma$ inducing factor, is produced from monocyte/macrophage, dendritic cell, or keratinocyte (19). As a representative cytokine mediating $\mathrm{T}_{\mathrm{H}} 1$ response, IL-18 stimulates IFN $\gamma$ production from $\mathrm{T}_{\mathrm{H}} 1$ cells and IL-12 is known to up-regulate IL-18 receptor expression on $\mathrm{T}_{\mathrm{H}} 1$ cells and $\mathrm{B}$ cells $(19,27)$. Our present study also demonstrated a significant downregulation of IL-18 mRNA expression in the skin tissues of mice treated with DNCB for atopic dermatitis induction (Fig. 2). Proinflammatory activity of IL-18 has been shown more culminating when inflammatory response approaches to chronic phase, resulting in predominance of $\mathrm{T}_{\mathrm{H}} 1$-mediated immune reactivities in chronic stage of atopic dermatitis $(3,28,29)$. Since the present murine model for atopic dermatitis occurrence is considered rather an acute induction model through two time sensitization and two time challenge with DNCB for 2 weeks, the suppression of IL18 mRNA transcript could be evoked by a systemic predominance of $\mathrm{T}_{\mathrm{H}} 2$ response during the atopic dermatitis induction in mice $(10,11,13)$. Meanwhile, it will not be guaranteed for similar downregulation of epidermal IL-18 protein expression as the mRNA expression. Many studies reported similar direction of IL-18 mRNA and protein expression in skin tissue $(30,31)$. Whereas counterregulation of IL-18 mRNA and protein expression was also reported during cutaneous wound repair in mice, in that skin IL-18 protein expression was significantly enhanced upon injury but IL-18 mRNA level was concomitantly decreased (32). The authors assumed that IL-18 mRNA transcripts might be degraded as IL-18 protein synthesis moved on.

$\mathrm{T}_{\mathrm{H}} 2$ cell is major source of IL-13 production in overall immune system. IL-13 directly acts on keratinocytes and leads to a disturbance of skin homeostasis through enhancement of inflammatory cell infiltration in human atopic dermatitis $(1,18,28)$. Concomitant up-regulation of both IL-4 and IL-13 has been extensively addressed in various immune phenomena related with the predominance of $\mathrm{T}_{\mathrm{H}} 2$ cell reactivities $(33,34)$. Furthermore, IL-13 exhibited reduction of FLG gene expression (3). In addition, IL-13 
is considered a key player for differentiation of naïve dendritic cell to DC2 phenotype (12). The increase in IL-13 production was demonstrated from axillary lymph node $\mathrm{T}$ cells in the mice with atopic dermatitis compared to the control mice in our murine model (10). But, IL-13 mRNA expression levels in the skin tissues were unexpectedly shown not much different among the groups (Fig. 2). Intraepithelial resident $\gamma \delta \mathrm{T}$ cells were recently reported as potent producers of IL-13 (35). Furthermore, a significant upregulation of IL-13 protein expression was demonstrated in subacute and chronic AD skin lesions (36). Considering these findings together, atopic dermatitis induced in our murine model may be in rather early stage, therefore activities of intraepithelial $\mathrm{T}$ cells were not much modulated. Concerning the functions of IL-13 together, no significant alteration of IL-13 mRNA expression in the skin tissues of mice with atopic dermatitis could result in the up-regulation of filaggrin protein instead of the reduced amounts of filaggrin protein.

Our present study adds some characteristic features to the published list of the human atopic dermatitis like immune or pathophysiological features in the murine model, in that lowered expression of skin IL-18 and TNF- $\alpha$ mRNA expression, cytokines involved with $\mathrm{T}_{\mathrm{H}} 1$ cell-mediated immune responses. Expression of filaggrin protein in skin tissues should be evaluated as time-dependent manner from acute stage to chronic stage in future. Overall, the present murine model is believed valuable for development of preventive or therapeutic medicinal products for atopic dermatitis.

\section{ACKNOWLEDGMENTS}

This study was proceeded as a part of thesis research of JiYoun Kim, and partially supported by Catholic University of Daegu (grant \#20121291).

Received September 19, 2017; Revised October 15, 2017; Accepted October 20, 2017

\section{REFERENCES}

1. Weidinger, S. and Novak, N. (2016) Atopic dermatitis. Lancet, 387, 1109-1122.

2. Deckers, I.A., McLean, S., Linssen, S., Mommers, M., van Schayck, C.P. and Sheikh, A. (2012) Investigating international time trends in the incidence and prevalence of atopic eczema 1990-2010: a systematic review of epidemiological studies. PLoS ONE, 7, e39803.

3. Egawa, G. and Weninger, W. (2015) Pathogenesis of atopic dermatitis: a short review. Cogent Biol., 1, 1103459.

4. Hensel, P., Santoro, D., Favrot, C., Hill, P. and Griffin, C. (2015) Canine atopic dermatitis: detailed guidelines for diagnosis and allergen identification. BMC Vet. Res., 11, 196.
5. Matsumoto, M., Ra, C., Kawamoto, K., Sato, H., Itakura, A., Sawada, J., Ushio, H., Suto, H., Mitsuishi, K., Hikasa, Y. and Matsuda, H. (1999) IgE hyperproduction through enhanced tyrosine phosphorylation of Janus kinase 3 in NC/ Nga mice, a model for human atopic dermatitis. J. Ітmиnol., 162, 1056-1063.

6. Konishi, H., Tsutsui, H., Murakami, T., Yumikura-Futatsugi, S., Yamanaka, K., Tanaka, M., Iwakura, Y., Suzuki, N., Takeda, K., Akira, S., Nakanishi, K. and Mizutani, H. (2002) IL-18 contributes to the spontaneous development of atopic dermatitis-like inflammatory skin lesion independently of IgE/stat6 under specific pathogen-free conditions. PNAS, 99, 11340-11345.

7. Lee, G.R. and Flavell, R.A. (2004) Transgenic mice which overproduces Th2 cytokines develop spontaneous atopic dermatitis and asthma. Int. Immunol., 16, 1150-1160.

8. Chan, L.S., Robinson, N. and Xu, L. (2001) Expression of interleukin-4 in the epidermis of transgenic mice results in a pruritic inflammatory skin disease: an experimental animal model to study atopic dermatitis. J. Invest. Dermatol., 117, 977-983.

9. Yoshioka, T., Hikita, I., Matsutani, T., Yoshida, R., Asakawa, M., Toyosaki-Maeda, T., Hirasawa, T., Suzuki, R., Arimura, A. and Horikawa, T. (2003) DS-Nh as an experimental model of atopic dermatitis induced by Staphylococcus aureus producing staphylococcal enterotoxin. Cell. Immunol., 108, 562-569.

10. Kim, S.-N., Kim, Y.-C., Kim, H.-A. and Heo, Y. (2011) A potential mechanism of 2,4-dinitrochlorobenzene-mediated immune alterations resembling human atopic dermatitis in mice. Quant. Bio-Sci., 30, 85-90.

11. Lee, S.H., Baek, S.J., Kim, H.A. and Heo, Y. (2006) 2,4Dinitrochlorobenzene-induced atopic dermatitis like immune alteration in mice. Toxicol. Res., 22, 357-364.

12. Cook, P.C., Jones, L.H., Jenkins, S.J., Wynn, T.A., Allen, J.E. and MacDonald, A.S. (2012) Alternatively activated dendritic cells regulate $\mathrm{CD} 4^{+}$T-cell polarization in vitro and in vivo. PNAS, 109, 9977-9982.

13. Lee, S.-H., Heo, Y. and Kim, Y.-C. (2010) Effect of German chamomile oil application on alleviating atopic dermatitislike immune alterations in mice. J. Vet. Sci., 11, 35-41.

14. Wu, Z., Hansmann, B., Meyer-Hoffert, U., Gläser, R. and Schröder, J.-M. (2009) Molecular identification and expression analysis of filaggarin-2, a member of the S100 fusedtype protein family. PLoS ONE, 4, e5227.

15. Heo, Y., Mondal, T.K., Gao, D., Kasten-Jolly, J., Kishikawa, H. and Lawrence, D.A. (2007) Posttranscriptional inhibition of interferon-gamma production by lead. Toxicol. Sci., 96, 92-100.

16. Chen, L., Segal, D.M. and Mash, D.C. (1999) Semi-quantitative reverse-transcriptase polymerase chain reaction: an approach for the measurement of target gene expression in human brain. Brain Res. Prot., 4, 132-139.

17. Cabanillas, B. and Novak, N. (2016) Atopic dermatitis and filaggrin. Curr. Opi. Immunol., 42, 1-8.

18. Matsunaga, M.C. and Yamauchi, P.S. (2016) IL-4 and IL-13 inhibition in atopic dermatitis. J. Drugs Dermatol., 15, 925929.

19. Dinarello, C.A., Novick, D., Kim, S. and Kaplanski, G. 
(2013) Interleukin-18 and IL-18 binding protein. Front. Immunol., 4, 289.

20. Bashir, M.M., Sharma, M.R. and Werth, V.P. (2009) TNFalpha production in skin. Arch. Dermatol. Res., 30, 87-91.

21. Brown, S.J. and Irwin McLean, W.H. (2012) One remarkable molecule: Filaggrin. J. Invest. Dermatol., 132, 751-762.

22. Thyssen, J.P. and Kezic, S. (2014) Causes of epidermal filaggrin reduction and their role in the pathogenesis of atopic dermatitis. J. Allergy Clin. Immunol., 134, 792-799.

23. Sandilands, A., Sutherland, C., Irvine, A.D. and Irwin McLean, W.H. (2009) Filaggrin in the frontline: role in skin barrier function and disease. J. Cell Sci., 122, 1285-1294.

24. Visser, M.J., Verberk, M.M., Campbell, L.E., Irwin Mclean, W.H., Calkoen, F., Bakker, J.G., van Dijk, F.J.H., Bos, J.D. and Kezic, S. (2013) Filaggrin los-of-function mutations and atopic dermatitis as risk factors for hand eczema in apprentice nurses: part II of a prospective cohort study. Contact Derm., 70, 139-150.

25. Furue, M., Tsuji, G., Mitoma, C., Nakahara, T., Chiba, T., Morino-Koga, S. and Uchi, H. (2015) Gene regulation of filaggrin and other skin barrier proteins via aryl hydrocarbon receptor. J. Dermatol. Sci., 80, 83-88.

26. Ye, Y.-M., Km, B.-Y., Shin, Y.-S., Park, H.-S. and Leung, D.Y.M. (2014) Increased epidermal filaggrin in chronic idiopathic urticarial is associated with severity of urticaria. Ann. Allergy Asthma Immunol., 112, 533-538.

27. Yoshimoto, T., Takeda, K., Tanaka, T., Ohkusu, K., Kashiwamura, S., Okamura, H., Akira, S. and Nakanishi, K. (1998) IL-12 up-regulates IL-18 receptor expression on T cells, Th1 cells, and B cells: synergism with IL-18 for IFN-g production. J. Immunol., 161, 3400-3407.

28. Wang, A. and Landen, N.X. (2015) New insights into T cells and their signature cytokines in atopic dermatitis. IUBMB, 67, 601-610.
29. Lee, J., Cho, D. and Park, H. (2015) IL-18 and cutaneous inflammatory diseases. Int. J. Mol.Sci., 16, 29357-29369.

30. Stoll, S., Müller, G., Kurimoto, M., Saloga, J., Tanimoto, T., Yamauchi, H., Okamura, H., Knop, J. and Enk, A.H. (1997) Production of IL-18 (IFN-gamma-inducing factor) messenger RNA and functional protein by murine keratinocytes. $J$. Immunol., 159, 298-302.

31. Gosmann, C., Frazer, I.H., Mattarollo, S.R. and Blumenthal, A. (2014) IL-18, but not IL-12, induces production of IFN-g in the immunosuppressive environment of HPV16 E7 transgenic hyperplastic skin. J. Invest. Dermatol., 134, 25622569.

32. Kämpfer, H., Kalina, U., Mühl, H., Pfeilschofter, J. and Frank, S. (1999) Counterregulation of interleukin-18 mRNA and protein expression during cutaneous wound repair in mice. J. Invets. Dermatol., 113, 369-374.

33. Heo, Y., Zhang, Y., Gao, D., Miller, V.M. and Lawrence, D.A. (2011) Aberrant immune responses in a mouse with behavioral disorders. PLOS ONE, 6, e20912.

34. Ravi, G., Heo, Y., Lim, G.-D., Song, E.-S., Roque, K., Lee, J.-H., Kim, Y.-G., Cho, A.-R., Shin, S.-J., Kim, C.-Y., Bang, G.-H., Bahng, J.-Y. and Kim, H.-A. (2017) Altered immune responses in broiler chicken husbandry workers and their association with endotoxin exposure. Ind. Health, doi: 10.2486/indhealth.2017-0049 [Epub ahead of print].

35. Dalessandri, T., Crawford, G., Hayes, M., Seoane, R.C. and Strid, J. (2016) IL-13 from intraepithelial lymphocytes regulates tissue homeostasis and protect against carcinogenesis in the skin. Nat. Commun., 7, 12080.

36. Oh, M.-H., Oh, S.-Y., Lu, J., Lou, H., Myers, A.C., Zhu, Z. and Zheng, T. (2013) TRPA1-dependent pruritis in IL-13induced chronic atopic dermatitis. J. Immunol., 191, 53715382. 